\title{
Avaliação da resistência à compressão do concreto pela substituição parcial do cimento Portland por cinzas do bagaço de cana-de-açúcar
}

Evaluation of the compressive strenght of concrete by partial replacement of Portland cement by sugarcane bagasse ash

Concreto evaluación resistencia a la compresion mediante la sustitución parcial del cemento Portland com cenizas de bagazo de caña 


\section{ANAP $B_{\text {rasil }}$}

\section{RESUMO}

Este trabalho sugere a substituição parcial do cimento Portland pela cinza do bagaço da cana-de-açúcar (CBC), dando uma destinação viável ao resíduo e contribuindo para redução do impacto ambiental causado pelas industrias cimenteiras. A cinza, que é composta basicamente por sílica, pode ser usada como adição mineral em argamassas e concretos. É importante salientar que os aspectos físicos e químicos da CBC podem variar de acordo com o processo da queima, por isso a importância de seu estudo. O procedimento experimental foi feito substituindo o cimento Portland no concreto em proporções de 15 e $25 \%$ e comparando a resistência à compressão com o concreto padrão, sem substituição aos 14 dias e 28 dias de cura. A pesquisa aponta que o concreto com substituição de $15 \%$ atingiu resistência à compressão de $18,67 \mathrm{MPa}$ aos 28 dias, possibilitando o uso. Porém, para trabalhos futuros, sugere-se que seja observada a resistência mecânica do concreto com CBC com um tempo de cura ainda maior, pois o material tem uma reação de hidratação mais retardada. Além de outros ensaios como fluorescência de raio $X$, e difração de raio $X$, para ser conhecido aspectos químicos do material.

PALAVRAS-CHAVE: Cinzas do bagaço de cana-de-açúcar. Cimento Portland. Desenvolvimento sustentável.

\section{ABSTRACT}

This work suggests the partial replacement of Portland cement by the sugarcane bagasse ash (BA), giving a viable destination to the waste and contributind to reduce the environmental impact caused by the cement indestries. The ash. which is basically composed by silica, can be used as mineral addition in mortars and concretes. It is important to note that the physical and chemical aspects of BA may vary according to the burning process, so is important it's study. The experimental procedure was done by relacing the Portland cement in proportion of 15 and $25 \%$ and comparing the compressive strenght with the standart concrete, without replacement at 14 days and 28 days of cure. The research indicates that the concrete with $15 \%$ replacement reached compressive strenth of $18,67 \mathrm{Mpa}$ at 28 days, allowing the use. However, for future works, it is suggested that the mechanical strength of the concrete with BA be observed with an even longer cure time because the material has a more delayed hydration reaction. In addition to others tests, such as X-ray florescence and X-ray diffraction, to be known chemical aspects of the material.

KEY WORDS: Sugarcane bagasse ashes. Portland cement. Sustainable development.

\section{RESUMEN}

Este trabajo sugiere la sustitución parcial cel cemento Portland por la ceniza del bagazo de la caña de azúcar (CBC), dando un destino viable al residuo y contribuyendo a la reducción del impacto ambiental causado por la industria del cement. La ceniza, que básicamente se compone de sílice, se puede utilizar como una adición mineral en morteros y hormigones. Es importante destacar que los aspectos físicos y químicos de la CBC pueden variar de acuerdo con el proceso de grabación, por lo que la importancia de su estudio. El procedimiento experimental se realizó mediante la sustitución del cemento Portland en el hormigón em proporciones de 15 y 25 \% y la resistencia a la compresión en comparación con el hormigón estándar sin sustitución después de 14 días y 28 días de curado. La investigación muestra que el hormigón con el reemplazo de 15\% alcanzó resistencia a la compresión de 18,67 MPa a los 28 días, lo que permite em uso. Sin embargo, para el trabajo futuro, se sugiere que la resistencia mecanica se observa hormigón que contiene $\mathrm{CBC}$ mayor tiempo una reacción de hidratación más retrasado. En otras pruebas, tales como fluorescencia de rayos $\mathrm{X}$ y difracción de rayos $\mathrm{X}$ a ser conocido aspectos químicos del material.

PALABRAS CLAVE: Las cenizas de bagazo de caña de azúcar. Cemento Portland. Desenvolvimiento sustentable. 


\section{Introdução}

A indústria do cimento é responsável por $3 \%$ das emissões mundiais de gases de efeito estufa (WORLD BUSINESS COUNCIL FOR SUSTAINABLE DEVELOPMENT- WBCSD, 2002) e por $5 \%$ das emissões de $\mathrm{CO}_{2}$, aproximadamente. Da emissão da indústria cimenteira, $50 \%$ referem-se ao processo produtivo do clínquer, cerca de $10 \%$ ao transporte e uso de eletricidade e os $40 \%$ restantes são proveniente da queima dos combustíveis para aquecimento dos fornos (CEMENT SUSTAINABILITY INITIATIVE - CSI, 2017). Estimativas apontam que a fabricação de cimento Portland em escala mundial são da ordem de 1,7 bilhões de toneladas por ano, o suficiente para produzir $6 \mathrm{~km}^{3}$ de concreto por ano ou $1 \mathrm{~m}^{3}$ por habitante (GARTNER, 2004). Já no Brasil, a produção em 2013 foi de 70,1 milhões de toneladas e a emissão específica de $\mathrm{CO}_{2}$ por tonelada de cimento é de $600 \mathrm{~kg}$ (Sindicato Nacional da Indústria do Cimento - SNIC, 2013).

Os impactos gerados pelo processo produtivo do cimento podem ocorrer em praticamente todas as suas fases, desde a extração, produção, até a sua disposição final, revelando o papel da indústria do cimento como elevado potencial poluidor. Há fontes de poluição em todas as etapas do processo - moagem e homogeneização das matérias-primas; clinquerização no forno rotativo; resfriamento do clínquer; moagem do clínquer; adições e produção de cimento, ensacamento e expedição; pontos de transferência de materiais (MAURY; BLUMENSCHEIN, 2012).

Os níveis de poluição e suas características não são os mesmos em todas as indústrias fabricantes de cimento, pois dependem das características tecnológicas e operacionais do processo industrial, em especial, dos fornos rotativos de clínquer, da composição química e mineralógica das matérias-primas e da composição química dos combustíveis empregados; da marcha operacional dos fornos de clínquer; e da eficiência dos sistemas de controle de emissão de poluentes instalados (SANTI; SEVÁ FILHO, 2004).

Os poluentes primários produzidos pelo processo de fabricação de cimento são: material particulado, dióxido de carbono, óxidos de carbono, óxidos e enxofre e óxidos de nitrogênio. De acordo com o órgão ambiental norte-americano - United States Environmental Protection Agency (USEPA)- , as plantas de fabricação de cimento estão entre as maiores fontes de emissão de poluentes atmosféricos perigosos, dos quais destacam-se as dioxinas e furanos; os metais tóxicos como mercúrio, chumbo, cádmio, arsênio, antimônio e cromo; os produtos de combustão incompleta e os ácidos halogenados (USA, 1991; USEPA, 1996, citado por SANTI;SEVÁ FILHO, 2004). Os metais pesados contidos das matérias-primas e combustíveis, mesmo em pequenas concentrações, devido ao comportamento físico-químico de seus compostos e sua volatilidade, podem ser emitidos na forma de material particulado ou de vapor através das chaminés das fábricas (USEPA, 2000, citado por SANTI;SEVÁ FILHO, 2004).

Foram estabelecidos padrões de emissão para material particulado, metais pesados, cloretos, monóxido de carbono, dioxinas e furanos, para o controle da poluição gerada nas plantas de fabricação de cimento. De uma forma geral, o material particulado proveniente dos fornos, moinhos e resfriador de clínquer é direcionado para chaminés e retido em coletores com ciclone, filtros de manga e precipitadores eletrostáticos. As medidas de controle para a 
redução da emissão de poeiras fugitivas nas áreas de mineração e na área industrial são o abatimento dos particulados por aspersão de água e o enclausuramento das áreas de estocagem e beneficiamento de materiais, com a instalação de sistemas exaustores e de filtros coletores de pós, além da pavimentação e da varrição de vias de circulação de veículos. $\mathrm{Na}$ maioria das plantas de clinquerização, entretanto, não são instalados equipamentos para o controle da emissão de gases de combustão, vapores de sais metálicos ou outras substâncias perigosas originadas no processo de clinquerizacão (SANTI; SEVA FILHO, 2004).

Além do risco ao meio ambiente, os trabalhadores da indústria cimenteira são prejudicados. De acordo com a pesquisa de Pinto Junior e Braga (2009), o processo de trabalho sofre algumas variações em cada cimenteira. Porém, há exposição aos resíduos em todas as etapas: recepção, coleta de amostra e análise físico-química, descarga, armazenamento, mistura, transporte interno, alimentação do sistema, limpeza de instalações e manuntenção de equipamentos. E mesmo sendo orientados ao uso dos equipamentos de proteção individual (EPI), os trabalhadores são expostos as substâncias tóxicas o que causa entre outras coisas dores de cabeça, náuseas, intoxicações agudas e crônicas, vômitos, cefaléia, vertigem e astenia, se o tóxico for absorvido pelo organismo. Mas se houver contato direto, pode causar irritações na pele, mucosa ocular, e vias respiratórias.

Visto todas essas dificuldades, desde o fim do século passado tem-se intensificado estudos para reduzir a utilização do cimento. Uma alternativa é a substituição do cimento por material pozolânico no concreto.

De acordo com a NBR 12653 (Associação Brasileira de Normas Técnicas - ABNT, 2012), materiais pozolânicos são materiais finos silicosos ou silicoaluminosos que, por si só não possuem atividade aglomerante ou pouca atividade, mas que na presença da água, reagem com o hidróxido de cálcio à temperatura ambiente formando compostos com propriedades cimentantes.

Malhotra e Mehta (1996) destacam alguns aditivos mineiras que são empregados atualmente no concreto, sendo eles a cinza volante, a escória de alto forno, a sílica ativa e a cinza da casca de arroz. A transformação de resíduos agroindustriais e industriais em produtos para o concreto pode ajudar a reduzir despesas e pacificar o impacto ambiental causado pela disposição e extração de matérias primas usadas para fabricação do clínquer. Além desses quesitos, é preciso destacar que a adição desses resíduos no concreto dá a ele vantagens em sua reologia, propriedade mecânica e durabilidade.

Outro material que vem sendo estudado como material pozolânico é a cinza do bagaço da cana- de- açucar ( $C B C)$. O Brasil conta com mais de nove milhões de hectares plantados de cana-de-açucar, sendo o estado de São Paulo o maior produtor, com mais de cinco milhões de hectares (Instituto Brasileiro de Geografia e Estatística- IBGE, 2017). Sendo que, na safra de 2015/16 foram produzidos 665,6 milhões de toneladas, com um crescimento de 4,9 \% em relação a safra do ano anterior (BRASIL, 2016). E, segundo Sales e Lima (2010), para cada uma tonelada de bagaço de cana-de-açúcar processada tem-se vinte e cinco quilos de CBC. Desta forma, o país apresenta um grande potencial de material. A co-geração com bagaço de cana- 


\section{ANAP Brasil}

\section{REVISTA CIENTIFICA 2017}

de-açúcar produz liberação sim de carbono na forma de $\mathrm{CO}_{2}$. Mas, o balanço nas emissões de $\mathrm{CO}_{2}$ é praticamente nula, pois, a biomassa queimada é resposta no ciclo seguinte da cultura da cana, através da fotossíntese (CORDEIRO, 2006).

Segundo Cordeiro et al. (2008), durante o processo da extração do caldo da cana-de-açúcar é gerado grande montante de bagaço (aproximadamente $30 \%$ da cana moída). 0 bagaço é queimado em caldeiras ( $95 \%$ de todo produzido no Brasil) para geração de vapor, assim, a cinza do bagaço da cana (CBC) é gerado, como resíduo, cujo destino pode causar problema ambiental. É constituída basicamente de sílica, $\mathrm{SiO}_{2}$, tendo assim potencial para ser usada como adição mineral em argamassas e concretos. A cinza é usada como fertilizantes nas plantações, mas ela não tem os nutrientes ideais para esse propósito (SALES; LIMA, 2010).

A tabela 1 apresenta os principais elementos químicos presentes na $C B C$ de três pesquisas (SINGH N., SINGH V., RAI 2000; Paula et al., 2008; CORDEIRO et al. ,2010).

Tabela 1: Elementos químicos das cinzas

\begin{tabular}{c|c|c|c} 
Elementos Quimicos & $\begin{array}{c}\text { SINGH N., SINGH V., } \\
\text { RAI (2000) }\end{array}$ & PAULA et al. (2009) & CORDEIRO et al. (2010) \\
\hline $\mathrm{SiO2}$ & 63,16 & 83,707 & 78,34 \\
\hline $\mathrm{Fe} 2 \mathrm{O} 3$ & 5,14 & 6,537 & 8,55 \\
\hline $\mathrm{Al} 2 \mathrm{O} 3$ & 9,7 & - & 3,61 \\
\hline $\mathrm{CaO}$ & 8,4 & 1,183 & 2,15 \\
\hline $\mathrm{MgO}$ & 2,9 & - & - \\
\hline $\mathrm{Na} 2 \mathrm{O}$ & - & - & 0,12 \\
\hline $\mathrm{K} 2 \mathrm{O}$ & - & 6,146 & 3,46 \\
\hline $\mathrm{TiO} 2$ & - & 1,162 & - \\
\hline $\mathrm{SO}$ & 2,87 & 0,682 & -
\end{tabular}

Singh N., Singh V., Rai (2000) fizeram substituições de 0,10,20 e 30\% do cimento Portland pela CBC . No resultado do teste de resistência à compressão, o resultado da amostra de $10 \%$ foi maior do que a sem substituição. Além disso nos testes de durabilidade e permeabilidade as amostras com a $\mathrm{CBC}$ se saíram melhor do que o padrão.

Paula et al. (2009) substituíram o cimento Portland pela CBC em argamassas em taxas de 0, 10, 20 e $30 \%$. Os resultados foram bastante satisfatórios, pois houve pouca perda de resistência em relação a argamassa sem substituição- a maior substituição, a de $30 \%$ houve somente $14 \%$ de perda-, mas para se alcançar maior resistência os pesquisadores sugerem a substituição de até $20 \%$.

Cordeiro et al. (2010) fizeram substituições do cimento Portland pela CBC em teores de 0, 10, 15 e $20 \%$. Os autores afirmam que as cinzas sejam moídas durante 120 minutos para que atinjam ao máximo sua atividade pozolânica, pois afirmam que há um aumento da reatividade da CBC em função da redução do tamanho médio das partículas que, por sua vez, é 


\section{ANAP Brasil}

\section{REVISTA CIENTIFICA 2017}

inversamente proporcional ao tempo de moagem. Assim, é possível a substituição de até $20 \%$, sem a perda das propriedades reológicas do concreto.

\section{Objetivos}

Avaliar a resistência à compressão dos concretos com substituição de 15 e $25 \%$ de cimento Portland por CBC, comparando com o concreto padrão, sem substituição.

\section{Metodologia}

\section{1 Materiais}

As cinzas foram coletadas de uma usina sucroalcooleira do município de Araçatuba, no interior do estado de São Paulo. O cimento utilizado foi o CP || E-32, que contém em sua composição a escória de alto forno, e os agregados usados foram a brita 1 e a areia grossa. Esses materiais foram obtidos em uma loja de materiais de construção.

\section{2 Traço}

Foram executados três traços diferentes, sendo um deles padrão e os outros dois com a cinza substituindo determinado percentual de cimento. Esses traços tem uma sequência lógica, cimento:areia:cinza do bagaço de cana-de-açúcar:brita:água, e a quantidade em massa de cada está listada na tabela 2.

\section{3 Ensaio de resistência à compressão}

Para avaliar a influência da substituição do CP pela CBC no concreto realizou-se o ensaio de resistência à compressão. Foram moldados corpos de prova cilíndricos com dimensões de 10 $\mathrm{cm}$ de diâmetro e $20 \mathrm{~cm}$ de altura e, para cada um dos traços moldaram-se três corpos de prova para cada uma das idades desejadas, sendo essas 14 (quatorze) e 28 (vinte e oito) dias, totalizando 18 (dezoito) corpos de prova. A tabela 2 resume as características da pesquisa.

\begin{tabular}{|c|c|c|c|c|c|c|c|}
\hline \multirow[t]{2}{*}{ Amostra } & \multirow[t]{2}{*}{ Traço } & \multirow{2}{*}{$\begin{array}{c}\text { Teor de } \\
\text { Subsitituição }\end{array}$} & \multirow{2}{*}{$\begin{array}{l}\text { Tipo de Corpo } \\
\text { de Prova }\end{array}$} & \multirow{2}{*}{$\begin{array}{l}\text { Corpo de } \\
\text { Prova (CP) }\end{array}$} & $\begin{array}{l}\text { Resistência Característisca } \\
\text { à Compressão (fck) - MPa }\end{array}$ & \multirow[t]{2}{*}{$\begin{array}{l}\text { Corpo de } \\
\text { Prova (CP) }\end{array}$} & \multirow{2}{*}{$\begin{array}{l}\text { Resistência Característisca } \\
\text { à Compressão (fck) - MPa } \\
28 \text { dias }\end{array}$} \\
\hline & & & & & 14 dias & & \\
\hline \multirow{4}{*}{ Padrão } & \multirow{4}{*}{ 1: $2,08: 0: 2,32: 0,55$} & \multirow{4}{*}{ - } & \multirow{4}{*}{ cilíndrico } & СР 01 & 28,59 & CP 04 & 30,88 \\
\hline & & & & $\mathrm{CP} 02$ & 29,72 & $\mathrm{CP} 05$ & 30,54 \\
\hline & & & & $\mathrm{CP} 03$ & 28,02 & CP 06 & 29,45 \\
\hline & & & & MÉDIA & 28,78 & MÉDIA & 30,29 \\
\hline \multirow{4}{*}{ СВ01 } & \multirow{4}{*}{$0,85: 2,08: 0,15: 2,32: 0,55$} & \multirow{4}{*}{$15 \%$} & \multirow{4}{*}{ cilíndrico } & CP 07 & 12,65 & CP 10 & 18,42 \\
\hline & & & & CP 08 & 15,85 & CP 11 & 18,37 \\
\hline & & & & CP 09 & 14,14 & CP 12 & 19,23 \\
\hline & & & & MÉDIA & 14,22 & MÉDIA & 18,67 \\
\hline \multirow{4}{*}{ CBO2 } & \multirow{4}{*}{$0,75: 2,08: 0,25,: 2,32: 0,55$} & \multirow{4}{*}{$25 \%$} & \multirow{4}{*}{ cilíndrico } & CP 13 & 11,85 & CP 16 & 15,95 \\
\hline & & & & CP 14 & 12,59 & CP 17 & 16,12 \\
\hline & & & & CP 15 & 12,29 & CP 18 & 16,21 \\
\hline & & & & MÉDIA & 12,24 & MÉDIA & 16,09 \\
\hline
\end{tabular}




\section{ANAP Brasil}

\section{REVISTA CIENTIFICA $\quad 2017$}

\section{Resultados}

A média da resistência dos corpos-de-prova está listado na tabela 3 e no gráfico 1.

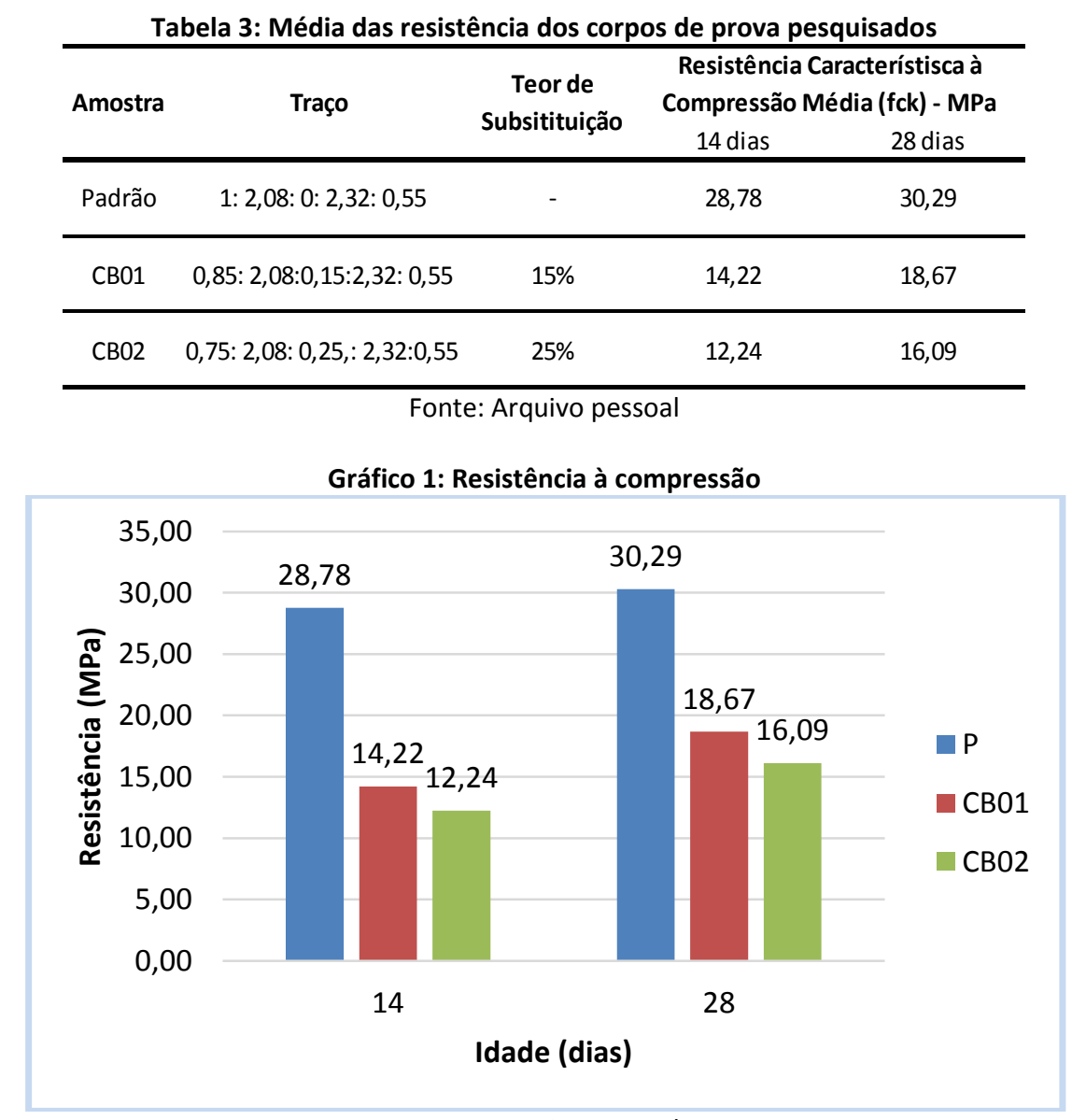

Fonte: Arquivo Pessoal

A partir do gráfico 1, analisando a resistência à compressão aos 14 dias de cura, verificou-se que o corpo de prova moldado com o concreto de traço padrão obteve a maior resistência , 28,78 MPa, seguido do corpo de prova moldado com o concreto de traço CB01, 14,22 MPa, e do corpo de prova moldado com o concreto de traço CB02, 12, $24 \mathrm{MPa}$ o qual obteve a menor resistência a compressão. Em termos percentuais, a redução de resistência em relação a amostra padrão foi de 50,59\% e 57,47\% para as amostras de traço CB01 E CB02.

Ainda de acordo com o gráfico 1, analisando a resistência à compressão das amostras com 28 dias de cura, a resistência atingida pelo concreto moldado com o traço padrão foi de 30,29 $\mathrm{MPa}$, já o concreto moldado com o traço $\mathrm{CB} 01$ foi de $18,67 \mathrm{MPa}$ e o concreto moldado com o traço CB02 foi de 16,09 MPa. Em termos percentuais a perda foi menor em relação ao concreto moldado com o traço padrão, de 38,36\% para o CB01; 53,11\% para o CB02.

É notável a queda da resistência dos corpos-de-prova com o aumento da substituição do cimento Portland pela $C B C$, porém foi observado que essa diferença diminui conforme o 


\section{ANAP Brasil}

\section{REVISTA CIENTIFICA $\quad 2017$}

tempo de cura do concreto aumenta, indicando que o material usado tem uma reação de hidratação ligeiramente retardada.

\section{Conclusão}

A cinza do bagaço de cana-de-açúcar é um importante material pozolânico, principalmente no Brasil, onde a cultura da cana-de-açúcar tem grande destaque. E apesar da queda de resistência nesta pesquisa, a CBC se mostrou como uma boa substituta parcial do cimento Portland, com melhores resultados na substituição de $15 \%$. Sugere-se em trabalhos futuros um tempo de cura maior dos concretos, para verificação da hidratação ligeiramente retardada do traço com a cinza.

Sugere-se também mais ensaios, como o de fluorescência de raio $X$, para saber a composição química do material, difração de raio $\mathrm{X}$, para tomar conhecimento da estrutura atômica do material, antes de aplicá-lo no concreto.

Considerando os dados do SNIC de 2013 sobre a produção de cimento e a emissão de $\mathrm{CO}_{2}$, a substituição de pelo menos $15 \%$ de cimento no concreto possibilitariam a redução de 6,3 milhões de toneladas de $\mathrm{CO}_{2}$ por ano no Brasil.

\section{Agradecimentos}

À Universidade Paulista - UNIP campus de Araçatuba, por ter cedido o laboratório de materiais de construção civil e aos familiares pelo imenso apoio durante toda a pesquisa.

\section{Referências Bibliográficas}

ASSOCIAÇÃO BRASILEIRA DE NORMAS TÉCNICAS. NBR 12653: Materiais Pozolânicos. Rio de Janeiro, 2012. 5 p.

BRASIL, Portal. Safra de cana 2016/17 cresce em produção e área. 2017. Disponível em: <http://www.brasil.gov.br/economia-e-emprego/2016/04/safra-de-cana-2016-17-cresce-em-producaoe-area>. Acesso em: 28 abr. 2017.

CEMENT SUSTAINABILITY INITIATIVE. $\mathbf{C O}_{2}$ and Climate Protection. Disponível em: <https://www.wbcsdcement.org/index.phd/key-issues/climate-protection>. Acesso em: 15 abr. 2017.

CORDEIRO, G. C. Utilização de cinzas ultrafinas do bagaço de cana-de-açúcar e da casca de arroz como aditivos minerais em concreto, Rio de Janeiro, 2006, Tese (Doutorado) COPPE, Universidade Federal do Rio de Janeiro, $445 \mathrm{p}$.

CORDEIRO, G. C. et al. Pozzolanic activity and filler effect of sugarcane bagasse ash in Portland cement and lime mortars. Cement and Concrete Composites, [s.I.], v. 30, n. 5, p. 410-418, maio 2008. Elsevier BV http://dx.doi.org/10.1016/j.cemconcomp.2008.01.001.

CORDEIRO, G.C.; TOLEDO FILHO, R.D.; FAIRBAIRN, E.M.R. Cinza ultrafina do bagaço de cana-de-açucar: material pozolânico de alto potencial para países tropicais. Revista Ibracon de Estruturas e Materiais, [s.l.], v. 3, n. 1, p. 50-67, mar. 2010. FapUNIFESP (SciELO). http://dx.doi.org/10.1590/s19834195010000100004.

GARTNER, E. Industrially interesting approaches to "low $\mathrm{CO}_{2}$ " cements. Cement and Concrete Research, [s.I], v. 34, n. 9, p. 1489-1498, set 2004. Elsevier BV. http://dx.doi.org.10.1016/j.cemconres.2004.01.021. 


\section{ANAP Brasil

\section{REVISTA CIENTIFICA 2017}

INSTITUTO BRASILEIRO DE GEOGRAFIA E ESTATISTICA. Levantamento sistemático de produção agrícola. 2017. Disponível em:<http://www.ibge.gov.br/home/estatistica/indicadores/ agropecuaria/Ispa/Ispa_2017. Acesso em: 28 abr. 2017.

MALHOTRA, V.M., MEHTA, P. K. 1996. Pozzolanic and cementitious materials, 1 ed. Amsterdam: Gordon and Breach Publishers, 1996. $191 \mathrm{p}$.

MAURY, M. B.; BLUMENSCHEIN, R. N. Produção de cimento: Impactos a saúde a ao meio ambiente. Sustentabilidade em Debate, Brasília, v.3, n. 1, p. 75-96, jun. 2012.

PAULA, M O. de et al. Potencial da cinza do bagaço da cana-de-açúcar como material de substituição parcial de cimento Portland. Revista Brasileira de Engenharia Agrícola e Ambiental, [s.l.], v. 13, n. 3, p. 353- 357, jun. 2009. Fap UNIFESP (SciELO). http://dx.doi.org/10.1590/s1415-43662009000300019.

SALES, A.; LIMA, S. A. Use of brazilian sugarcane bagasse ash in concrete as sand replacement. Waste Management, [s.l.] v. 30, n. 6, p. 1114-1122, jun. 2010. Elsevier BV. http://dx.doi.org/10.1016/j.wasman,2010.01.026

SANTI, A. M. M.; SEVÁ FILHO, A. O. Combustíveis e riscos ambientais na fabricação de cimento : casos na Região do Calcário ao Norte de Belo Horizonte e possíveis generalizações. In: Encontro da ANPPAS Associação Nacional de Pós-Graduação e Pesquisa em Ambiente e Sociedade, 2., 2004, Piracicaba. Anais... Encontro da ANPPAS, 2004. p.1-18. Disponível em: < http://www.repositorio.ufop.br/bitstream/ 123456789/1295/1/EVENTO_Combust\%C3\% ADveisRiscosAmbientais.pdf >. Acesso em: 20 abr. 2017.

SINGH, N. B; SINGH, V. D; RAI, S. Hydration of bagasse ash-blended Portland cement. Cement and Concrete Research, [s.I.],v. 30, n. 9, p. 1485-1488, set. 2000. Elsevier BV. http://dx.doi.org/10.1016/s0008-8846(00)00324-0.

SINDICATO NACIONAL DA INDÚSTRIA DO CIMENTO (Rio de Janeiro). Relatório Anual 2013. 2013. Disponível em:<http://www.snic.org.br/pdf/RelatorioAnual2013final.pdf>. Acesso em: 10 mar. 2017.

PINTO JÚNIOR, A. G.; BRAGA, A. M. C. B. Trabalho e saúde: a atividade da queima de resíduos tóxicos em fornos de cimenteiras de Cantagalo, Rio de Janeiro. Ciência e saúde coletiva, [s.l], v. 14, n. 6, p.20052014, dez. 2009. FapUNIFESP (sciELO). http://dx.doi.org/10,1590/s1413-81232009000600008.

WORLD BUSINESS COUNCIL FOR SUSTAINABLE DEVELOPMENT. Toward a sustainable cement industry. Mar.2002. Disponível em: < https://www.wbcsdcement.org/pdf/battelle/Toward \%20a\%20sustainable\%20cement\%20industry.pdf>. Acesso em 05 abr. 2017. 